\title{
On Janowski Type Harmonic Meromorphic Functions with respect to Symmetric Point
}

\author{
Muhammad Ghaffar Khan $\left(D,{ }^{1}\right.$ Bakhtiar Ahmad $\left(D,{ }^{2}\right.$ Maslina Darus $\left(D,{ }^{3}\right.$ \\ Wali Khan Mashwani $\mathbb{D}^{1}{ }^{1}$ and Shahid Khan ${ }^{1}$ \\ ${ }^{1}$ Institute of Numerical Sciences, Kohat University of Science and Technology, Kohat, Pakistan \\ ${ }^{2}$ Govt. Degree College Mardan, 23200 Mardan, Pakistan \\ ${ }^{3}$ Department of Mathematical Sciences, Faculty of Science and Technology, Universiti Kebangsaan Malaysia, 43600 Bangi, \\ Selangor, Malaysia
}

Correspondence should be addressed to Maslina Darus; maslina@ukm.edu.my

Received 21 November 2020; Revised 17 December 2020; Accepted 21 January 2021; Published 27 January 2021

Academic Editor: Sivasubramanian Srikandan

Copyright (c) 2021 Muhammad Ghaffar Khan et al. This is an open access article distributed under the Creative Commons Attribution License, which permits unrestricted use, distribution, and reproduction in any medium, provided the original work is properly cited.

In this paper, we define a new class of Sakaguchi type-meromorphic harmonic functions in the Janowski domain that are starlike with respect to symmetric point. Furthermore, we investigate some important geometric properties like sufficiency criteria, distortion bound, extreme point theorem, convex combination, and weighted means.

\section{Introduction and Definitions}

One of the contemporary developments in Mathematics is the solicitations of harmonic analysis in other fields. Like various other fields, it has immensely influenced and nurtured the branch of geometric function theory. Jahangiri et al. [1] defined and studied a subclass of harmonic and univalent functions. Another example of such work would be an article of Porwal and Dixit [2], who used a certain convolution operator involving hypergeometric functions to define a class of univalent functions. As a consequence, many mathematicians generalized many ideas of this field and various important results with the help of some operators; the work of Porwal et al. [3], Porwal et al. [4], and Porwal and Dixit [5] are worth mentioning here. Recently, some subclasses of harmonic functions were investigated by Arif et al. [6] and Khan et al. [7]. To start with, we give preliminaries which will be useful in understanding the concepts of this research.
A real-valued function $u(x, y)$ is said to be harmonic in a domain $\mathbb{D} \subset \mathbb{C}$ if it has a continuous second partial derivative and satisfy the Laplace's equation

$$
\frac{\partial^{2} u}{\partial x^{2}}+\frac{\partial^{2} u}{\partial y^{2}}=0
$$

A continuous complex-valued function $f=u+i v$ is said to be harmonic in a complex domain $\mathbb{U}$ if both its real and imaginary parts are real harmonic in $\mathbb{U}$. In any simply connected domain $\mathbb{U} \subset \mathbb{C}$, one can write $f=h+\bar{g}$, where $h$ and $g$ are analytic in $\mathbb{U}$. The class of such functions is denoted by $\mathscr{H}$. The condition $\left|h^{\prime}(z)\right|>\left|g^{\prime}(z)\right|$ is necessary and sufficient for $f$ to be locally univalent and sense preserving in $\mathbb{U}$, see [8]. There are different papers on univalent harmonic functions defined in unit disc $\mathbb{D}=\{z:|z|<1\}$, for details, see [9-14]. For $z \in \mathbb{D}^{*}=\mathbb{D} \backslash\{0\}$, in the punctured open unit disc and let $\mathscr{M}_{\mathscr{H}}$ denote the class of functions 


$$
f(z)=h(z)+g \overline{(z)}=\frac{1}{z}+\sum_{n=1}^{\infty} a_{n} z^{n}+\sum_{n=1}^{\infty^{-}} b_{n} z^{n}
$$

which are harmonic in $\mathbb{D}^{*}$ where $h$ is analytic in $\mathbb{D}^{*}$ and has a simple pole at the origin with residue 1 , while $g$ is analytic in $\mathbb{D}$. The class $\mathscr{M}_{\mathscr{H}}$ was studied in [15-17]. Furthermore, denoted by $\mathscr{M}_{\mathscr{H}}$, a subclass of $\mathscr{M}_{\mathscr{H}}$, consisting a functions of the form

$$
f(z)=h(z)+g \overline{(z)}=\frac{1}{z}+\sum_{n=1}^{\infty}\left|a_{n}\right| z^{n}-\sum_{n=1}^{\infty}\left|b_{n}\right| \bar{z}^{n}
$$

which are harmonic univalent in punctured unit disc $\mathbb{D}^{*}$.

For functions $f \in \mathscr{M}_{\mathscr{H}}$ given by (2) and $F \in \mathscr{M}_{\mathscr{H}}$ given by

$$
F(z)=H(z)+G \overline{(z)}=\frac{1}{z}+\sum_{n=1}^{\infty} A_{n} z^{n}+\sum_{n=1}^{\infty^{-}} B_{n} z^{n}
$$

we recall the Hadamard product (or convolution) of $f$ and $F$ by

$$
(f * F)(z)=\frac{1}{z}+\sum_{n=1}^{\infty} a_{n} A_{n} z^{n}+\sum_{n=1}^{\infty} \bar{b}_{n} B_{n} z^{n}\left(z \in \mathbb{D}^{*}\right) .
$$

In terms of the Hadamard product (or convolution), we choose $F$ as a fixed function in $\mathscr{H}$ such that $(f * F)($ $z$ ) exists for any $f \in \mathscr{H}$, and for various choices of $F$, we get different linear operators which have been studied in the recent past.

Recently, Khan et al. [18] introduced and studied a class of meromorphic starlike functions with respect to symmetric point in circular domain i.e.,

$$
-\frac{2 z f^{\prime}(z)}{f(z)-f(-z)} \prec \frac{1+A z}{1+B z}
$$

Motivated from the above discussion on harmonic functions and class of meromorphic starlike functions with respect to symmetric point, we introduced the class of meromorphic harmonic univalent functions as:

Let $-1 \leq B<A \leq 1$. Then, the function $f \in \mathscr{M}_{\mathscr{H}}$ is in the class $\mathscr{M}_{\mathscr{H}}^{* *}[A, B]$ if it satisfies the condition

$$
-\frac{2 \mathscr{D}_{\mathscr{H}} f(z)}{f(z)-f(-z)} \prec \frac{1+A z}{1+B z},(z \in \mathbb{D}),
$$

where the symbol " $\prec "$ represent well-known subordination and

$$
\mathscr{D}_{\mathscr{H}} f(z)=z h^{\prime}(z)-\overline{z g}^{\prime}(z) \text {, }
$$

or equivalently

$$
\left|\frac{\mathscr{D}_{\mathscr{H}} f(z)+(f(z)-f(-z) / 2)}{B_{\mathscr{H}} f(z)+A(f(z)-f(-z) / 2)}\right|<1,(z \in \mathbb{D}) .
$$

Furthermore, we denote $\mathscr{M}_{\mathscr{H}}^{* *}[A, B]$ subclass of $\mathscr{M}_{\mathscr{H}}^{* *}[A$, $B]$ consisting of harmonic meromorphic functions $f=h+\bar{g}$ of the form (3).

\section{Main Results}

Theorem 1. Let $f=h+\bar{g}$ be of the form (2) and satisfies the condition

$$
\sum_{n=1}^{\infty} \alpha_{n}\left|a_{n}\right|+\beta_{n}\left|b_{n}\right| \leq 1
$$

with

$$
\begin{aligned}
& \alpha_{n}=\frac{\left|(1+B) n+(1+A)\left(1-(-1)^{n} / 2\right)\right|}{A-B} \text { and } \\
& \beta_{n}=\frac{\left|(1+B) n-(1+A)\left(1-(-1)^{n} / 2\right)\right|}{A-B},
\end{aligned}
$$

then $f$ is harmonic univalent sense-preserving in $\mathbb{D}^{*}$ and $f \in$ $\mathscr{M}_{\mathscr{H}}^{* *}[A, B]$.

Proof. For $0<\left|z_{1}\right| \leq\left|z_{2}\right|<1$, we obtain

$$
\begin{aligned}
&\left|\frac{f\left(z_{1}\right)-f\left(z_{2}\right)}{h\left(z_{1}\right)-h\left(z_{2}\right)}\right| \geq 1-\left|\frac{g\left(z_{1}\right)-g\left(z_{2}\right)}{h\left(z_{1}\right)-h\left(z_{2}\right)}\right| \\
&=1-\left|\frac{z_{1} z_{2} \sum_{n=1}^{\infty} b_{n}\left(z_{1}^{n}-z_{2}^{n}\right)}{\left(z_{1}-z_{2}\right)-z_{1} z_{2} \sum_{n=1}^{\infty} a_{n}\left(z_{1}^{n}-z_{2}^{n}\right)}\right| \\
&>1-\frac{\sum_{n=1}^{\infty} n\left|b_{n}\right|}{1-\sum_{n=1}^{\infty} n\left|a_{n}\right|}>1-\frac{\sum_{n=1}^{\infty} \beta_{n}\left|b_{n}\right|}{1-\sum_{n=1}^{\infty} \alpha_{n}\left|a_{n}\right|} \geq 0,
\end{aligned}
$$

where we have used (10) and this shows that the function is univalent.

Now to show $f(z)$ is sense-preserving harmonic mapping in $\mathbb{D}^{*}$, consider

$$
\begin{aligned}
\left|h^{\prime}(z)\right| & \geq \frac{1}{|z|^{2}}-\sum_{n=1}^{\infty} n\left|a_{n}\right||z|^{n-1} \geq 1-\sum_{n=1}^{\infty} \alpha_{n}\left|a_{n}\right| \geq \sum_{n=1}^{\infty} \beta_{n}\left|b_{n}\right| \\
& \geq \sum_{n=1}^{\infty} n\left|b_{n}\right| \geq\left|g^{\prime}(z)\right| .
\end{aligned}
$$

This shows that $f$ is sense-preserving.

Now, to show that $f \in \mathscr{M}_{\mathscr{H}}^{* *}[A, B]$ from (9), it is enough to show that

$$
\left|\mathscr{D}_{\mathscr{H}} f(z)+\frac{f(z)-f(-z)}{2}\right|-\left|B \mathscr{D}_{\mathscr{H}} f(z)+A \frac{f(z)-f(-z)}{2}\right|<0 .
$$


For this, consider

$$
\begin{aligned}
\mid \mathscr{D}_{\mathscr{H}} f & (z)+\frac{f(z)-f(-z)}{2}|-| B \mathscr{D}_{\mathscr{H}} f(z)+A \frac{f(z)-f(-z)}{2} \mid \\
= & \left|\sum_{n=1}^{\infty}\left[\left(n+\frac{1-(-1)^{n}}{2}\right) a_{n} z^{n}+\left(n+\frac{1-(-1)^{n}}{2}\right) b_{n} z^{n}\right]\right|-\mid \frac{A-B}{z} \\
& +\sum_{n=1}^{\infty}\left[\left(B n+A \frac{1-(-1)^{n}}{2}\right) a_{n} z^{n}-\left(B n+A \frac{1-(-1)^{n}}{2}\right) b_{n} z^{n}\right] \mid \\
\leq & \sum_{n=1}^{\infty}\left[\left(n+\frac{1-(-1)^{n}}{2}\right)\left|a_{n}\right||z|^{n}+\left(n+\frac{1-(-1)^{n}}{2}\right)\left|b_{n}\right||z|^{n}\right] \\
& -\frac{A-B}{|z|} \sum_{n=1}^{\infty}\left[\left(B n+A \frac{1-(-1)^{n}}{2}\right)\left|a_{n}\right||z|^{n}+\left(B n+A \frac{1-(-1)^{n}}{2}\right)\left|b_{n}\right||z|^{n}\right] \\
\leq & \sum_{n=1}^{\infty}\left\{\left|(1+B) n+(1+A) \frac{1-(-1)^{n}}{2}\right|\left|a_{n}\right||z|^{n}+\mid(1+B) n\right. \\
& +\left.(1+A) \frac{1-(-1)^{n}}{2}|| b_{n}|| z\right|^{n}-\frac{A-B}{|z|} \\
= & \frac{A-B}{|z|} \sum_{n=1}^{\infty}\left[\frac{\left|(1+B) n+(1+A)\left(1-(-1)^{n} / 2\right)\right|}{A-B}\left|a_{n}\right||z|^{n+1}\right. \\
& \left.+\frac{\left|(1+B) n+(1+A)\left(1-(-1)^{n} / 2\right)\right|}{A-B}\left|b_{n}\right||z|^{n+1}\right]-\frac{A-B}{|z|} \\
< & \frac{A-B}{|z|}\left\{\sum_{n=1}^{\infty}\left[\alpha_{n}\left|a_{n}\right|+\beta_{n}\left|b_{n}\right|\right]-1\right\}<0 .
\end{aligned}
$$

Hence, complete the proof.

Example 2. The meromorphic univalent function

$$
f(z)=\frac{1}{z}+\sum_{n=1}^{\infty} \frac{x_{n}}{\alpha_{n}} z^{n}+\sum_{n=1}^{\infty} \frac{y_{n}}{\beta_{n}} \bar{z}^{n}
$$

such that $\sum_{n=1}^{\infty}\left(\left|x_{n}\right|+\left|y_{n}\right|\right)=1$, we have

$$
\sum_{n=1}^{\infty}\left(\alpha_{n}\left|a_{n}\right|+\beta_{n}\left|b_{n}\right|\right)=\sum_{n=1}^{\infty}\left(\left|x_{n}\right|+\left|y_{n}\right|\right)=1 .
$$

Thus, $f \in \mathscr{M}_{\mathscr{H}}^{* *}[A, B]$ and above coefficient bound given in (10) is sharp for this function.

Theorem 3. Let $f=h+\bar{g} \in \mathscr{M}_{\bar{H}}$ and of the form (3), then $f \in \mathscr{M}_{\mathscr{H}}^{* *}[A, B]$ if it satisfies the condition

$$
\sum_{n=1}^{\infty} \alpha_{n}\left|a_{n}\right|+\beta_{n}\left|b_{n}\right| \leq 1
$$

with

$$
\begin{aligned}
& \alpha_{n}=\frac{\left|(1+B) n+(1+A)\left(1-(-1)^{n} / 2\right)\right|}{A-B} \text { and } \\
& \beta_{n}=\frac{\left|(1+B) n-(1+A)\left(1-(-1)^{n} / 2\right)\right|}{A-B} .
\end{aligned}
$$

Theorem 4. Let $f=h+\bar{g} \in \mathscr{M}_{\mathscr{H}}^{* *}[A, B]$ and of the form (3), $0<|z|=r<1$. Then,

$$
\frac{1}{r}-\frac{A-B}{2+A+B} r \leq|f(z)| \leq \frac{1}{r}+\frac{A-B}{2+A+B} r .
$$

Proof. Consider

$$
\begin{aligned}
|f(z)| & =\left|\frac{1}{z}+\sum_{n=1}^{\infty}\left(a_{n} z^{n}+b_{n}^{-} z^{n}\right)\right| \leq \frac{1}{r}+\sum_{n=1}^{\infty}\left(\left|a_{n}\right|+\left|b_{n}\right|\right) r^{n} \\
& \leq \frac{1}{r}+r \sum_{n=1}^{\infty}\left(\left|a_{n}\right|+\left|b_{n}\right|\right) \leq \frac{1}{r}+\frac{A-B}{2+A+B} r .
\end{aligned}
$$

Similarly, proceeding as above we get

$$
\begin{aligned}
|f(z)| & =\left|\frac{1}{z}+\sum_{n=1}^{\infty}\left(a_{n} z^{n}+b_{n}^{-} z^{n}\right)\right| \geq \frac{1}{r}-r \sum_{n=1}^{\infty}\left(\left|a_{n}\right|+\left|b_{n}\right|\right) \\
& =\frac{1}{r}-\frac{A-B}{2+A+B} r .
\end{aligned}
$$

Hence, this completes the result.

Theorem 5. Let $f=h+\bar{g}$, and of the form (3) then $f \in \mathscr{M}_{\mathscr{H}}^{* *}$ $[A, B]$ if and only if

$$
f(z)=\sum_{n=0}^{\infty}\left(\gamma_{n} h_{n}+\delta_{n} g_{n}\right)
$$

where

$$
\begin{aligned}
& h_{0}(z)=\frac{1}{z}, h_{n}(z)=\frac{1}{z}+\frac{A-B}{\left|(1+B) n+(1+A)\left(1-(-1)^{n} / 2\right)\right|} z^{n}, n=1,2, \cdots \\
& g_{0}(z)=\frac{1}{z}, g_{n}(z)=\frac{1}{z}-\frac{A-B}{\left|(1+B) n+(1+A)\left(1-(-1)^{n} / 2\right)\right|} z^{n}, n=1,2, \cdots,
\end{aligned}
$$

where $1 \geq \gamma_{n} \geq 0,1 \geq \delta_{n} \geq 0$, and $\sum_{n=0}^{\infty}\left(\gamma_{n}+\delta_{n}\right)=1$.

Proof. Let

$$
\begin{aligned}
f(z)= & \gamma_{0} h_{0}+\delta_{0} g_{0}+\sum_{n=1}^{\infty}\left(\gamma_{n} h_{n}+\delta_{n} g_{n}\right) \\
= & \left(\gamma_{0}+\delta_{0}\right) \frac{1}{z}+\sum_{n=1}^{\infty} \gamma_{n}\left(\frac{1}{z}+\frac{A-B}{\left|(1+B) n+(1+A)\left(1-(-1)^{n} / 2\right)\right|} z^{n}\right) \\
& +\sum_{n=1}^{\infty} \delta_{n}\left(\frac{1}{z}-\frac{A-B}{\left|(1+B) n+(1+A)\left(1-(-1)^{n} / 2\right)\right|} z^{n}\right), \\
= & \sum_{n=1}^{\infty}\left(\gamma_{n}+\delta_{n}\right) \frac{1}{z}+\sum_{n=1}^{\infty} \gamma_{n} \frac{A-B}{\left|(1+B) n+(1+A)\left(1-(-1)^{n} / 2\right)\right|} z^{n} \\
& -\sum_{n=1}^{\infty} \delta_{n} \frac{A-B}{\left|(1+B) n+(1+A)\left(1-(-1)^{n} / 2\right)\right|} z^{n} .
\end{aligned}
$$

Proof. The proof is similar to Theorem 1, so omitted. 
Thus,

$$
\begin{aligned}
\sum_{n=1}^{\infty}\left\{\alpha_{n}\left(\frac{A-B}{\left|(1+B) n+(1+A)\left(1-(-1)^{n} / 2\right)\right|} \gamma_{n}\right)\right. \\
\left.\quad+\beta_{n}\left(\frac{A-B}{\left|(1+B) n+(1+A)\left(1-(-1)^{n} / 2\right)\right|} \delta_{n}\right)\right\} \\
=\sum_{n=1}^{\infty}\left(\gamma_{n}+\delta_{n}\right)=1-\gamma_{0}-\delta_{0} \leq 1,
\end{aligned}
$$

hence, $f \in \mathscr{M}_{\mathscr{H}}^{* *}[A, B]$. Conversely, let $f \in \mathscr{M}_{\mathscr{\mathscr { H }}}^{* *}[A, B]$. Set

$$
\begin{aligned}
\gamma_{n} & =\frac{\left|(1+B) n+(1+A)\left(1-(-1)^{n} / 2\right)\right|}{A-B}\left|a_{n}\right|, 0 \leq \gamma_{n} \leq 1, \delta_{n} \\
& =\frac{\left|(1+B) n+(1+A)\left(1-(-1)^{n} / 2\right)\right|}{A-B}\left|b_{n}\right|, 0 \leq \delta_{n} \leq 1, \gamma_{0} \\
& =1-\sum_{n=1}^{\infty} \gamma_{n}-\sum_{n=1}^{\infty} \delta_{n} .
\end{aligned}
$$

Therefore, $f$ can be written as

$$
\begin{aligned}
f(z)= & \frac{1}{z}+\sum_{n=1}^{\infty}\left|a_{n}\right| z^{n}-\sum_{n=1}^{\infty}\left|b_{n}\right| \bar{z}^{n} \\
= & \frac{1}{z}+\sum_{n=1}^{\infty} \frac{A-B}{\left|(1+B) n+(1+A)\left(1-(-1)^{n} / 2\right)\right|} \gamma_{n}\left|a_{n}\right| z^{n} \\
& -\sum_{n=1}^{\infty} \frac{A-B}{\left|(1+B) n+(1+A)\left(1-(-1)^{n} / 2\right)\right|} \delta_{n}\left|b_{n}\right| z^{n} \\
= & \frac{1}{z}+\sum_{n=1}^{\infty} \frac{A-B}{\left|(1+B) n+(1+A)\left(1-(-1)^{n} / 2\right)\right|} \gamma_{n}\left|a_{n}\right| z^{n} \\
& -\sum_{n=1}^{\infty} \frac{A-B}{\left|(1+B) n+(1+A)\left(1-(-1)^{n} / 2\right)\right|} \delta_{n}\left|b_{n}\right| z^{n} \\
= & \left(\gamma_{0}+\delta_{0}\right) \frac{1}{z}+\sum_{n=1}^{\infty} \gamma_{n}\left(\frac{1}{z}+\frac{A-B}{\left|(1+B) n+(1+A)\left(1-(-1)^{n} / 2\right)\right|} z^{n}\right) \\
& +\sum_{n=1}^{\infty} \delta_{n}\left(\frac{1}{z}-\frac{A-B}{\left|(1+B) n+(1+A)\left(1-(-1)^{n} / 2\right)\right|} z^{n}\right) \\
= & \sum_{n=1}^{\infty}\left(\gamma_{n} h_{n}+\delta_{n} g_{n}\right), \quad \text { hence required. }
\end{aligned}
$$

Theorem 6. The class $\mathscr{M}_{\mathscr{H}}^{* *}[A, B]$ is closed under convex combination.

Proof. For $k \in \mathbb{N}$, let $f_{k} \in \mathscr{M}_{\mathscr{H}}^{* *}[A, B]$, be of the form

$$
f_{k}(z)=\frac{1}{z}+\sum_{n=1}^{\infty}\left(\left|a_{k, n}\right| z^{n}-\left|b_{k, n}\right| z^{n}\right)
$$

Then form (10), we get

$$
\sum_{n=1}^{\infty}\left(\alpha_{n}\left|a_{k, n}\right|+\beta_{n}\left|b_{k, n}\right|\right) \leq 1
$$

For $\sum_{k=1}^{\infty} \delta_{k}=1,\left(0 \leq \delta_{k} \leq 1\right)$, the convex combination of $f_{k}$ is

$$
\sum_{k=1}^{\infty} \delta_{k} f_{k}(z)=\frac{1}{z}+\sum_{n=1}^{\infty}\left(\sum_{k=1}^{\infty} \delta_{k}\left|a_{k, n}\right|\right) z^{n}-\sum_{n=1}^{\infty}\left(\sum_{k=1}^{\infty} \delta_{k}\left|b_{k, n}\right|\right) \overline{z^{n}} .
$$

Using (10), we have

$$
\begin{aligned}
& \sum_{n=1}^{\infty}\left(\alpha_{n} \sum_{k=1}^{\infty} \delta_{k}\left|a_{k, n}\right|+\beta_{n} \sum_{k=1}^{\infty} \delta_{k}\left|b_{k, n}\right|\right) \\
& =\sum_{k=1}^{\infty} \delta_{k}\left[\sum_{n=1}^{\infty}\left(\alpha_{n}\left|a_{k, n}\right|+\beta_{n}\left|b_{k, n}\right|\right)\right] \leq \sum_{k=1}^{\infty} \delta_{k}=1,
\end{aligned}
$$

thus prove our desired results.

Theorem 7. Let $f_{k} \in \mathscr{M}_{\mathscr{H}}^{* *}[A, B]$, for $k=\{1,2\}$ be of the form (29), then, their weighted $F_{i}$ mean is also in the class $\mathscr{M}_{\mathscr{H}}^{* *}[A, B]$, where $F_{i}$ is defined below

$$
F_{i}(z)=\frac{(1-i) f_{1}(z)+(1+i) f_{2}(z)}{2} .
$$

Proof. From (33), one may easily write

$$
\begin{aligned}
F_{i}(z)= & \frac{1}{z}+\frac{(1-i) f_{1}(z)+(1+i) f_{2}(z)}{2} \\
= & \frac{1}{z}+\sum_{k=1}^{\infty}\left[\frac{(1-j)\left|a_{n, 1}\right|+(1+j)\left|a_{n, 2}\right|}{2} z^{n}\right. \\
& \left.-\frac{(1-j)\left|b_{n, 1}\right|+(1+j)\left|b_{n, 2}\right|}{2} z^{n}\right] .
\end{aligned}
$$

To show that $F_{i} \in \mathscr{M}_{\mathscr{\mathscr { H }}}^{* *}[A, B]$, it is enough to show that

$$
\begin{gathered}
\sum_{k=1}^{\infty}\left[\left|\frac{(1-j)\left|a_{n, 1}\right|+(1+j)\left|a_{n, 2}\right|}{2}\right| \alpha_{n}\right. \\
\left.+\left|\frac{(1-j)\left|b_{n, 1}\right|+(1+j)\left|b_{n, 2}\right|}{2}\right| \beta_{n}\right] \leq 1 .
\end{gathered}
$$

Now consider

$$
\begin{aligned}
\sum_{k=1}^{\infty} & {\left[\left|\frac{(1-j)\left|a_{n, 1}\right|+(1+j)\left|a_{n, 2}\right|}{2}\right| \alpha_{n}+\left|\frac{(1-j)\left|b_{n, 1}\right|+(1+j)\left|b_{n, 2}\right|}{2}\right| \beta_{n}\right] } \\
& =\sum_{k=1}^{\infty}\left[\frac{(1-j)\left|a_{n, 1}\right| \alpha_{n}+(1-j)\left|b_{n, 1}\right| \beta_{n}}{2}+\frac{(1+j)\left|a_{n, 2}\right| \alpha_{n}+(1+j)\left|b_{n, 2}\right| \beta_{n}}{2}\right] \\
& =\frac{(1-j)}{2} \sum_{k=1}^{\infty}\left(\left|a_{n, 1}\right| \alpha_{n}+\left|b_{n, 1}\right| \beta_{n}\right)+\frac{(1+j)}{2} \sum_{k=1}^{\infty}\left(\left|a_{n, 2}\right| \alpha_{n}+\left|b_{n, 2}\right| \beta_{n}\right) \\
& \leq \frac{(1-j)}{2}+\frac{(1+j)}{2}=1 .
\end{aligned}
$$

Hence, $F_{i} \in \mathscr{M}_{\mathscr{H}}^{* *}[A, B]$. 


\section{Data Availability}

Data used to support the findings of this study are included within the article.

\section{Conflicts of Interest}

The authors declare that they have no competing interests.

\section{Authors' Contributions}

All authors jointly worked on the results and they read and approved the final manuscript.

\section{Acknowledgments}

The third author is supported by UKM Grant: GUP-2019032 .

\section{References}

[1] J. M. Jahangiri, G. Murugusundaramoorthy, and K. Vijaya, "Salagean-type harmonic univalent functions," Southwest Journal of Pure and Applied Mathematics, vol. 2, pp. 77-82, 2002.

[2] S. Porwal and K. K. Dixit, "An application of certain convolution operator involving hypergeometric functions," The Rajasthan Academy of Physical Sciences, vol. 9, no. 2, pp. 173-186, 2010.

[3] S. Porwal, K. K. Dixit, A. L. Pathak, and R. Agarwal, "A subclass of harmonic univalent functions with positive coefficients defined by generalized Salagean operator," The Rajasthan Academy of Physical Sciences, vol. 11, no. 2, pp. 93-102, 2012.

[4] S. Porwal, K. K. Dixit, V. Kumar, and P. Dixit, "On a subclass of analytic functions defined by convolution," General Mathematics, vol. 19, no. 3, pp. 57-65, 2011.

[5] S. Porwal and K. K. Dixit, "New subclasses of harmonic starlike and convex functions," Kyungpook National University, vol. 53, pp. 467-478, 2013.

[6] M. Arif, O. Barkub, H. M. Srivastava, S. Abdullah, and S. A. Khan, "Some Janowski type harmonic q-starlike functions associated with symmetrical points," Mathematics, vol. 8, no. 4, p. 629, 2020.

[7] M. G. Khan, B. Ahmad, and T. Abdeljawad, “Applications of a differential operator to a class of harmonic mappings defined by Mittag-Leffer functions," Aims Mathematics, vol. 5, no. 6, pp. 6782-6799, 2020.

[8] T. Shiel-Small, "Constants for planar harmonic mappings," Journal of the London Mathematical Society, vol. 2, no. 2, pp. 237-248, 1990.

[9] M. G. Khan, B. Ahmad, and N. E. Cho, Properties of Harmonic Mappings Associated with Polylogrithm FunctionsApplied Mathematics E-Notes.

[10] O. P. Ahuja, "Planar harmonic univalent and related mappings," Journal of Inequalities in Pure and Applied Mathematics, vol. 6, no. 4, pp. 1-18, 2005.

[11] H. Silverman, "Harmonic univalent functions with negative coefficients," Journal of Mathematical Analysis and Applications, vol. 220, no. 1, pp. 283-289, 1998.

[12] J. Dziok, “On Janowski harmonic functions," Journal of Applied Analysis, vol. 21, no. 2, pp. 99-107, 2015.
[13] S. Elhaddad, H. Aldweby, and M. Darus, "On a subclass of harmonic univalent functions involving a new operator containing q-Mittag-Leffler function," The International Journal of Mathematics and Computer Science, vol. 23, no. 4, pp. 833-847, 2019.

[14] J. M. Jahangiri, "Coefficient bounds and univalence criteria for harmonic functions with negative coefficients," Annales Universitatis Mariae Curie-Sklodowska, sectio AA, vol. 52, no. 2, pp. 57-66, 1998.

[15] H. Aldweby and M. Darus, "A new subclass harmonic meromorphic functions involving quantum calculus," Journal of classical analysis, vol. 6, pp. 152-162, 2015.

[16] R. M. El-Ashwah and B. A. Frasin, "Hadamard product of certain harmonic univalent meromorphic functions," Theory and Applications of Mathematics and Computer Sciences, vol. 5, pp. 126-131, 2015.

[17] J. M. Jahangiri and H. Silverman, "Harmonic meromorphic univalent functions with negative coefficients," Bulletin of the Korean Mathematical Society, vol. 36, pp. 763-770, 1999.

[18] M. G. Khan, M. Darus, B. Ahmad, G. Murugusundaramoorty, R. Khan, and N. Khan, "Meromorphic starlike functions with respect to symmetric points," International Journal of Analysis and Applications, vol. 18, pp. 1037-1047, 2020. 